Yépez Campoverde, J., Jiménez Calderón, L., Vázquez Hoehne, A. (2018): "Propuesta de catálogo normalizado de objetos aeronáuticos para el Ecuador”, GeoFocus (Artículos), $n^{\circ}$ 22, p. 21-42. ISSN: $1578-5157$ http://dx.doi.org/10.21138/GF.479

\title{
PROPUESTA DE CATÁlOGO NORMALIZADO DE OBJETOS AERONÁUTICOS PARA EL ECUADOR
}

\author{
JOSÉ YÉPEZ CAMPOVERDE ${ }^{1}$, LOLA JIMÉNEZ CALDERÓN², ANTONIO VÁZQUEZ \\ HOEHNE $^{2}$ \\ ${ }^{1}$ INSTITUTO ESPACIAL ECUATORIANO \\ Av. Emperador Carlos V Oe5-61 y Av. De la Prensa, 170511, Quito, Ecuador \\ jose.yepez@institutoespacial.gob.ec \\ ${ }^{2}$ UNIVERSIDAD POLITÉCNICA DE MADRID \\ Autovía de Valencia km 7, E28031 Madrid, España \\ ¿imenezloly@yahoo.com, antonio.vazquez@upm.es
}

RESUMEN

Uno de los aspectos clave para facilitar que la información se pueda intercambiar y compartir, asegurando que el significado de los contenidos se entienda de la misma manera por cualquier sistema, es la interoperabilidad semántica, cuya importancia puede ser significativa en campos como el aeronáutico. Para alcanzarla, se ha planteado la conformación de un catálogo normalizado de objetos o entidades, que contiene información descriptiva de una abstracción de estas entidades en el mundo real. Este artículo presenta una propuesta de catálogo de objetos aeronáuticos para el Ecuador a partir del análisis comparativo de tipos de objetos considerados en varios catálogos normalizados y la revisión de la normativa establecida para este fin. Con la ayuda de expertos de información aeronáutica, se ha conseguido por una parte, cumplir con la normativa vigente $\mathrm{y}$, a su vez, adaptarla al contexto nacional.

Palabras clave: catálogo de objetos, catálogo de entidades, estándar DFDD, estándar AIFDD, interoperabilidad semántica.

\section{STANDARD CATALOGUE OBJECTS AERONAUTICAL FOR ECUADOR}

\section{ABSTRACT}

A key to enable that information can be exchanged and shared, ensuring that meaning of the content is understood in the same way by any means, is the semantic interoperability, whose 


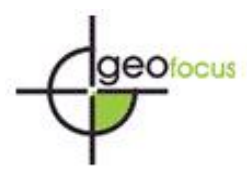

Yépez Campoverde, J., Jiménez Calderón, L., Vázquez. Hoehne, A. (2018): "Propuesta de catálogo normalizado de objetos aeronáuticos para el Ecuador”, GeoFocus (Artículos), $n^{\circ}$ 22, p. 21-42. ISSN: 1578-5157 http://dx.doi.org/10.21138/GF.479

importance can be significant in areas such as aeronautics. To achieve this kind of interoperability a methodological strategy consisting in a catalogue of objects or features was used as, which contains descriptive information about an abstraction of these feature in the real world. This article presents a proposal of a catalogue for Ecuador aircraft objects from the comparative analysis of object types considered in various standard catalogues and the revision of the regulations established for this purpose. With the help of experts in aeronautical information it was possible, on one hand, to comply with regulations and, on the other hand, to adapt it to the national context.

Keywords: object catalogue, feature catalogue, DFDD standard, AIFDD standard, semantic interoperability.

\section{Introducción}

La creciente relevancia de la interoperabilidad semántica proviene en buena medida de la necesidad de que la información se pueda intercambiar y compartir, asegurando que el significado de sus contenidos se entienda de la misma manera por cualquier sistema. Una estrategia para lograr este tipo de interoperabilidad, aunque de manera inicial, es el catálogo de objetos, que se concibe como una forma ampliamente utilizada de organizar, dentro de una taxonomía lógica, los conceptos que describen a las entidades u objetos del mundo real.

Uno de los problemas habituales entre los usuarios de información geográfica que más recursos consume, es el intercambio de datos e información. Las dificultades para que éstos se puedan compartir, aparecen fundamentalmente en los formatos, en los modelos y en las políticas de uso (Mas et al., 2012), pero también en la comprensión que se pueda alcanzar del significado estos datos geográficos. Los catálogos que describen estos tipos de objetos son indispensables para convertir los datos en información utilizable y, al proporcionar una mejor comprensión del contenido y el significado de los datos, promueven la difusión, el intercambio y el uso de los datos geográficos de forma estándar. En la medida que los proveedores y usuarios de datos geográficos tengan una comprensión compartida de los tipos de entidades del mundo real que representan los datos, serán capaces de juzgar si los datos suministrados son adecuados para su propósito (ISO, 2005).

Uno de los primeros ejemplos de catálogo normalizado a nivel internacional, es el Feature and Atribute Coding Catalogue (FACC), presentado por el Digital Geographic Information Working Group (DGIWG) al Comité Técnico de la ISO TC211 como proyecto de norma ISO en 1999. Mientras la propuesta de norma fue evolucionando, también el catálogo FACC fue realizando cambios y se fueron publicando nuevas versiones. Sin embargo, en el año 2013 el FACC fue reemplazado de forma definitiva por el DGIWG Feature Data Dictionary DFDD también del mismo grupo de trabajo DGIWG, y es el estándar reconocido actualmente (ver figura 1). Este catálogo se presenta como un ejemplo de implementación del esquema de diccionario de tipos de objeto geográficos y abarca 14 temáticas que corresponden a las categorías: Industria y Servicios, Geografía Socio-económica, Transportación, Hidrografía y Oceanografía, Fisiografía, Biota, Demarcación, Aeronáutica, Militar, Agua y Clima, Características, Nombres y Designaciones, Metadatos y Referencias, e Información Abstracta. 


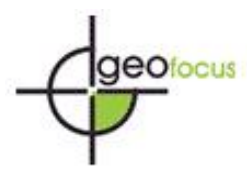

Yépez Campoverde, J., Jiménez Calderón, L., Vázquez Hoehne, A. (2018): "Propuesta de catálogo normalizado de objetos aeronáuticos para el Ecuador”, GeoFocus (Artículos), $n^{\circ}$ 22, p. 21-42. ISSN: $1578-5157$ http://dx.doi.org/10.21138/GF.479

Ya en el ámbito específico aeronáutico, el DGIWG publicó paralelamente en el año 2008, el Registro de Información Aeronáutica AIFDD (del inglés Aeronautical Information FDD Register), que identifica conceptos armonizados del Modelo de intercambio de Información Aeronáutica (AIXM), que también considera el fichero aeronáutico digital de vuelo (DAFIF), así como productos de la comunidad aeronáutica de uso común.

De modo general, a pesar de que una normativa pretende establecer directrices para todos los estamentos de su ámbito de aplicación, y su relevancia está más que justificada, su implementación efectiva no siempre resulta sencilla y en muchos casos idónea. En el caso de Ecuador, los catálogos internacionales disponibles no responden de forma total a la naturaleza y características de las actividades aeronáuticas del país, en consecuencia, el objetivo de este estudio fue proponer un catálogo para el Ecuador en este ámbito, a partir de otros catálogos normalizados, pero con una perspectiva hacia los requerimientos nacionales, de esta forma, se presenta inicialmente un análisis comparativo de tipos de objetos aeronáuticos considerados en diferentes catálogos normalizados y con el criterio de expertos de información aeronáutica, se presenta finalmente una propuesta de catálogo de tipos de objetos aeronáuticos enmarcado en las normas ISO 19126, ISO 19135 e ISO 19110 y en aportes de organismos rectores de actividades aeronáuticas como OACI y EUROCONTROL.

\section{Características de la Información Aeronáutica}

Los datos aeronáuticos, organizados y procesados para la toma de decisiones, componen la información aeronáutica, fundamental en el desarrollo de las operaciones aéreas y en la capacitación aeronáutica de tripulaciones, técnicos y controladores (Moya et al., 2010). Esta información en forma general, se publica como cartografía aeronáutica, diseñada para representar gráficamente las necesidades operativas de la aviación, y la información necesaria para cumplir el objetivo principal de la navegación aérea: "completar una ruta prefijada, cumpliendo las normas de circulación aérea establecidas y garantizando en todo momento la seguridad de los pasajeros" (Sáenz y Portillo, 2003). En este contexto cartográfico aparecen las cartas aeronáuticas con notable relevancia, ya que se convierten en fuentes completas y actualizadas, y constituyen el medio adecuado para suministrar información aeronáutica de manera fácil, concisa y coordinada, para el aseguramiento de las operaciones aéreas. Por lo tanto, resulta esencial disponer en todo momento de cartas cuya fuente sea completa, actualizada y autorizada (Calvo, 2003; Pérez et al., 2013). Resulta imprescindible también gestionar esta información aeronáutica bajo los mismos estándares de información geográfica, para poder articular, compartir y comprender esta información.

En un contexto mundial, la Organización de Aviación Civil Internacional (OACI), desde 1944, es la agencia especializada de las Naciones Unidas encargada de la aviación civil internacional y, en tal virtud, promueve reglamentos y normas únicas, para el desarrollo seguro y sostenible de la aviación civil mundial a través de la cooperación de sus estados miembros (Bravo et al., 2010). Para este fin, la OACI ha buscado de alguna manera delinear directrices para que la cartografía aérea se estandarice, en vista de lo cual, promulgó y exigió el cumplimiento del anexo 4 "Cartografía Aeronáutica" de las normas de aviación civil. Este documento que estandariza la presentación y publicación de la información cartográfica, ha dejado latente un problema de estandarización y armonización de la cartografía aeronáutica entre países, por cuanto cada país posee, según la propia norma de la OACI, autonomía para elaborar y actualizar su cartografía aérea 


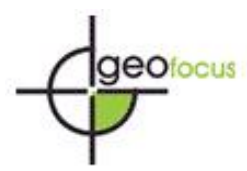

Revista Internacional de Ciencia y Tecnología de la Información Geográfica

International Review of Geographical Information Science and Technology

Yépez Campoverde, J., Jiménez Calderón, L., Vázquez Hoehne, A. (2018): "Propuesta de catálogo normalizado de objetos aeronáuticos para el Ecuador”, GeoFocus (Artículos), $n^{\circ}$ 22, p. 21-42. ISSN: 1578-5157 http://dx.doi.org/10.21138/GF.479

en base a sus realidades y necesidades. Como consecuencia, actualmente existen 21 tipos de cartas aeronáuticas clasificadas en 4 grupos de acuerdo a su utilización principal (Moya et al., 2010; Aguilar et al., 2009). Esta aparente falta de estandarización, derivada de las prioridades de cada país miembro de la OACI, no permite alcanzar la interoperabilidad de la información aeronáutica. Como resultado, a día de hoy, las operaciones aéreas que se llevan a cabo a nivel mundial, requieren que los pilotos y tripulaciones, tengan que analizar profundamente tipos de objetos geográficos y simbología diferente con cada cambio de espacio aéreo.

En vista de esta problemática, existen organismos que se han encargado de estandarizar la gestión de información aeronáutica. Programas regionales como el europeo Single European Sky ATM Research (SESAR) (EUROCONTROL, 2010; 2011) o la estrategia estadounidense liderada por la Administración Federal de Aviación (FAA) Next Generation Air Transportation System (NextGEN) (FAA, 2012) se enfocan particularmente en el modelado conceptual de los elementos de la información aeronáutica, en la estandarización de formatos de intercambio, (OGC, 2011) y en el fundamento tecnológico de los servicios integrados que, de forma enlazada, pueden lograr una gestión eficiente de la información aeronáutica.

Con el contexto anteriormente señalado, es primordial contar con información aeronáutica a nivel local, organizada y estructurada bajo los estándares internacionales que la rigen, y que a su vez, pueda extrapolarse hacia ámbitos de alcance regional y global.

\section{Marco Normativo para Catálogos de Tipos de Objeto}

La norma ISO 19110:2005 Geographic information -- Methodology for feature cataloguing define la metodología para catalogar tipos de entidades, especificando la forma de organización y divulgación de los objetos del mundo real en un conjunto de datos geográficos (Ariza y Rodríguez, 2008). Esta norma define, en su anexo B, los elementos obligatorios, condicionales u opcionales que debe tener un catálogo, así como la forma adecuada de caracterizar un tipo de objeto. También muestra, en su anexo C, ejemplos de aplicación de la norma, generalmente del diccionario FACC de DIGEST, y en el anexo A, se presentan las pruebas de conformidad (STANAG, 1998; Ariza y Pascual, 2008). Esta norma específica, entre otros aspectos, que cada tipo de objeto, tipos de atributo, asociación de objetos y operaciones en un catálogo, deben tener un nombre único en el catálogo, e incluir también definiciones y descripciones.

La norma ISO 19135:2005 Geographic information -- Procedures for item registration especifica los procedimientos que se deben seguir en el establecimiento y los registros de publicación de identificadores únicos, inequívocos y permanentes, y los significados que se asignan a los elementos de información geográfica, para lo cual, esta norma especifica los elementos de información necesarios para proporcionar la identificación y sentido a los objetos geográficos, así como para gestionar su registro (ISO, 2005). De forma general, se pueden registrar distintas clases de objetos especificados en normas técnicas, tales como las desarrolladas por ISO/TC 211 (Latingeo, 2009).

La norma ISO 19126 ha ido evolucionando en los últimos años como ISO 19126 Geographic information - Profile FACC data dictionary, desde la propuesta hecha por el grupo DGIWG al Comité Técnico TC/211, su aceptación en etapa preparatoria (WD) en 2001, borrador de comité (CD) en 2006, aprobación como borrador de norma (DIS) en el año 2007, borrador final de norma internacional (FDIS) en el 2008, y finalmente, en el año 2009 como norma internacional en 


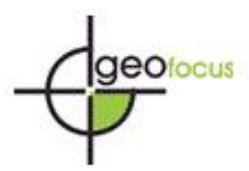

Yépez Campoverde, J., Jiménez Calderón, L., Vázquez Hoehne, A. (2018): "Propuesta de catálogo normalizado de objetos aeronáuticos para el Ecuador”, GeoFocus (Artículos), $n^{\circ}$ 22, p. 21-42. ISSN: 1578-5157 http://dx.doi.org/10.21138/GF.479

fase de publicación (IS/TS) con el nombre ISO 19126:2009 Geographic information -- Feature concept dictionaries and registers, como se la conoce actualmente (ver figura 1). Esta norma proporciona un esquema para la conformación de un diccionario de tipos de objeto geográfico y especifica procedimientos para el desarrollo y mantenimiento de registros de información geográfica. Éstos están en conformidad con la norma ISO 19135 (ISO, 2009). Además como aspecto relevante, esta norma incluye en su anexo D, el catálogo DFDD.

Por su parte, el grupo de trabajo DGIWG creó el estándar DIGEST (Digital Geographic Information Exchange Standard) que en su parte 4, incluyó el diccionario FACC mencionado anteriormente. Este diccionario en sus versiones 2.0 y 2.1 continuó formando parte del DIGEST, sin embargo de manera independiente el FACC continuó realizando mejora; tal es el caso que, en octubre de 2003, publicó una versión BL que incorpora tres enmiendas a la versión 2.1. Las versiones disponibles del catálogo FACC son: FACC 2.0 Enmienda 1: febrero 1999; FACC 2.1: septiembre de 2000; FACC BL 2003-4: octubre 2003.

El diccionario FACC, junto con el estándar DIGEST, han sido históricamente documentados como un producto analógico, sin embargo, en sus últimos años han sido publicados como un conjunto de archivos de Adobe Acrobat PDF. A pesar de los esfuerzos realizados por el grupo de trabajo por obtener una nueva versión del FACC, en el año 2013 fue declarado como extinto y en su reemplazo se publicó el DFDD también mencionado anteriormente. Este dejo de formar parte del estándar DIGEST y se incluyó como parte del anexo D de la norma ISO 19126:2009 (ver figura 1).

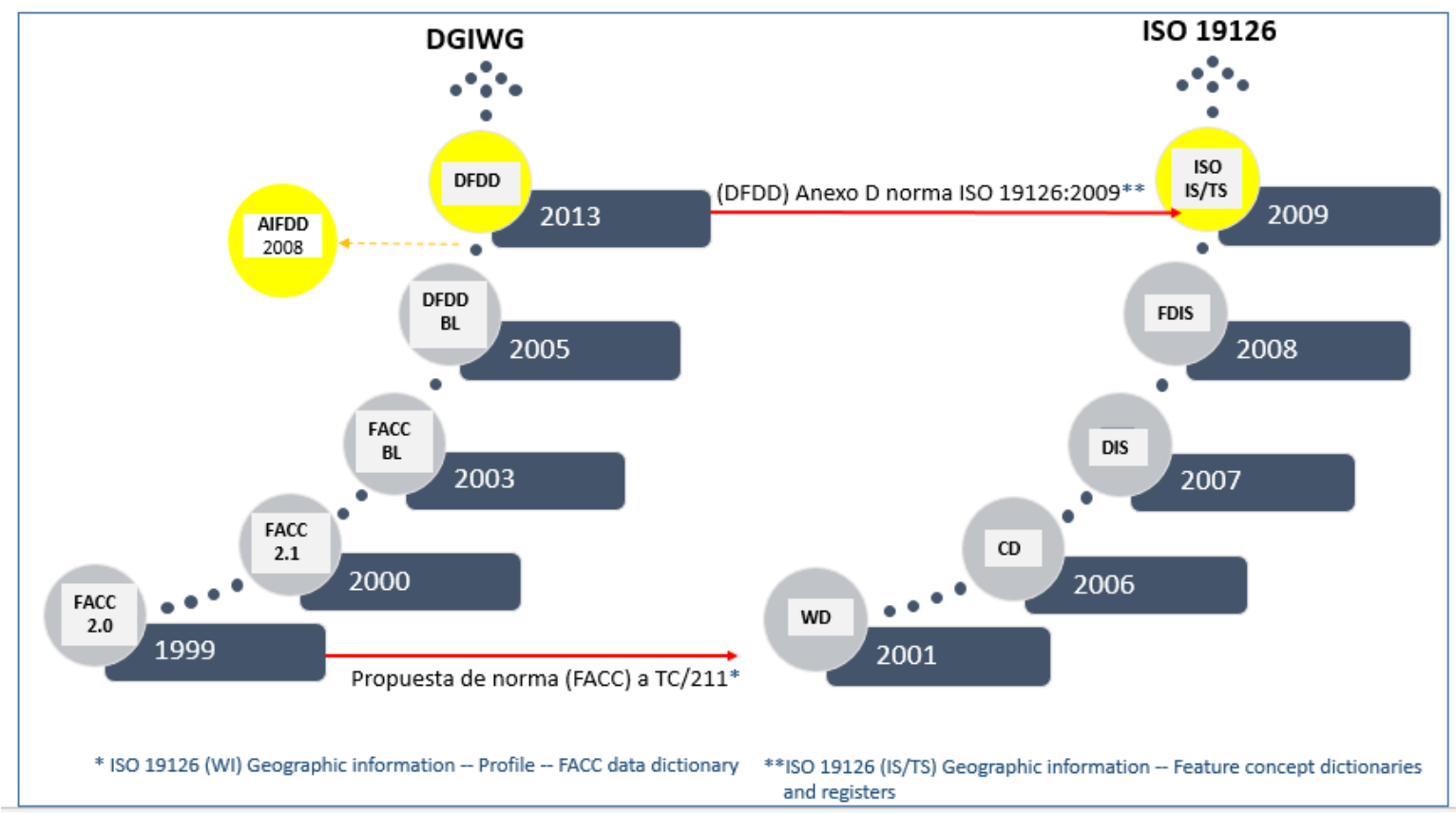

Figura 1. Evolución y relación de los catálogos del DGIWG y de la norma ISO 19126 
Yépez Campoverde, J., Jiménez Calderón, L., Vázquez Hoehne, A. (2018): "Propuesta de catálogo normalizado de objetos aeronáuticos para el Ecuador”, GeoFocus (Artículos), $n^{\circ} \quad 22, \quad$. 21-42. ISSN: 1578-5157 http://dx.doi.org/10.21138/GF.479

\section{Materiales, datos y métodos}

El estudio partió de una recopilación bibliográfica sobre normativa relacionada y catálogos con contenidos aeronáuticos a nivel nacional e internacional; a partir de esto se seleccionó la información idónea para realizar un análisis comparativo. En vista de que no se observó una importante disponibilidad de literatura específica sobre catálogos de tipos de objetos aeronáuticos, se consideró la participación de expertos en este tema, con el fin de analizar la aplicabilidad de estos estándares internacionales en el ámbito nacional ecuatoriano y en la conformación del catálogo de tipos de objetos aeronáuticos para el Ecuador. En la figura 2 se observa un esquema general del enfoque planteado en este estudio, donde se parte inicialmente de un análisis comparativo de catálogos en cuanto a estructura, tipos de objeto y semántica, posteriormente, con la ayuda de expertos, los objetos seleccionados se clasifican y codifican en las subcategorías respectivas. De esa forma se obtiene el catálogo final que se presenta en una plantilla diseñada para brindar facilidad de uso.

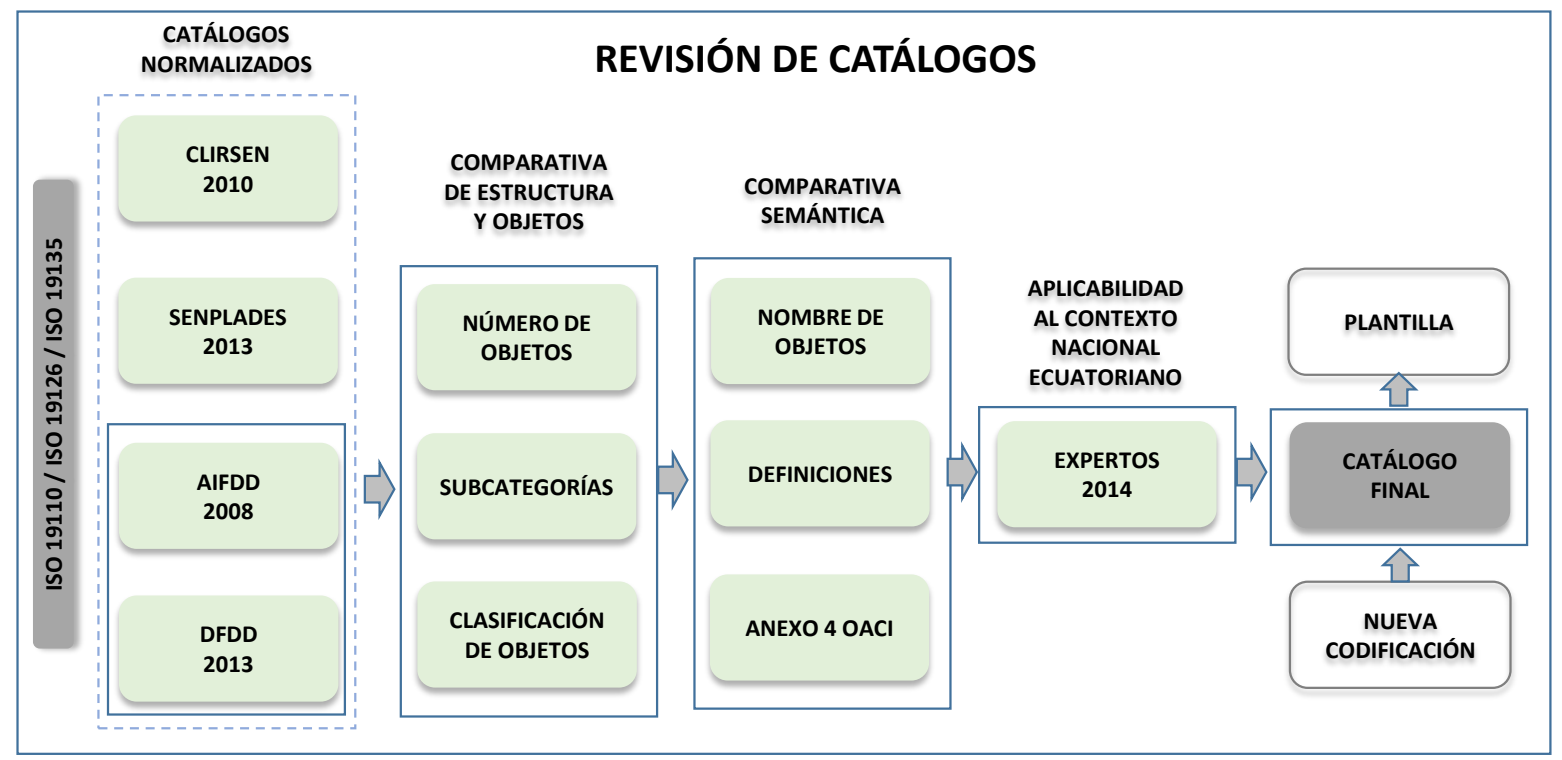

Figura 2. Enfoque planteado para la elaboración del catálogo propuesto

\subsection{Revisión de Catálogos}

En el contexto nacional, se revisaron dos catálogos de objetos disponibles; por una parte el Catálogo de Objetos Nacional, publicado en el año 2013 por la SENPLADES (Secretaría Nacional de Planificación y Desarrollo del Ecuador), en el cual se observan 23 tipos de objeto aeronáuticos ubicados en la categoría Infraestructura de Transporte y a su vez, clasificados en dos subcategorías; Transporte Aéreo y Ayudas para la Navegación. En este catálogo se observa que, a pesar de que se toman varios tipos de objetos, categorías y subcategorías del diccionario FACC y del diccionario DFDD, tal como se expresa en su publicación, no se encuentra normalizado en su totalidad. 
Yépez Campoverde, J., Jiménez Calderón, L., Vázquez Hoehne, A. (2018): "Propuesta de catálogo normalizado de objetos aeronáuticos para el Ecuador”, GeoFocus (Artículos), $n^{\circ}$ 22, p. 21-42. ISSN: $1578-5157$ http://dx.doi.org/10.21138/GF.479

Se revisó también el Catálogo de Objetos Temáticos del ex CLIRSEN (Centro de Levantamientos Integrados de Recursos Naturales por Sensores Remotos) publicado en su primera versión en el año 2010 y que sirvió como base para el Catálogo de Objetos Nacional anteriormente mencionado. Este catálogo temático, en su primera versión, utilizó un enfoque sencillo; inicialmente se recopilaron los tipos de objeto presentes en el estándar FACC, en su segunda versión del año 2000. A estos tipos se añadieron las categorías, subcategorías, tipos de objeto, atributos y dominios que no estaban considerados en este estándar y que presentaban relevancia para las principales instituciones generadoras y usuarias de información a nivel nacional, respetando la codificación del estándar FACC. En relación a la información aeronáutica, este catálogo seleccionó la categoría "Información Aeronáutica" presente en el FACC y la subcategoría "Aeródromos" también presente en el diccionario. En esta subcategoría se consideraron únicamente los tipos de objetos: aeropuerto, helipuerto y pista de aterrizaje.

En el contexto internacional se analizaron dos catálogos. Por una parte, el estándar específico para Información Aeronáutica AIFDD actualizado al año 2008; catálogo que identifica conceptos armonizados del Modelo de Intercambio de Información Aeronáutica (AIXM), -en el que intervienen la FAA, OACI, OGC y EUROCONTROL-, también considera el fichero aeronáutico digital de vuelo (DAFIF), y productos de la comunidad aeronáutica de uso común.

Por otra parte, se analizó el estándar más general DFDD, actualizado al año 2013 y que abarca 14 temáticas, entre ellas la Aeronáutica.

\subsection{Análisis comparativo}

En vista de que los dos catálogos analizados en el contexto nacional, generados por SENPLADES y ex CLIRSEN correspondientemente, son aplicaciones derivadas tanto del FACC como del DFDD y contienen un número mínimo de objetos aeronáuticos en relación al AIFDD y DFDD, se ha estimado oportuno considerar en el análisis comparativo, únicamente los catálogos internacionales AIFDD y DFDD. Estos estándares, ambos del DGIWG, presentan la misma estructura. El DFDD se constituye en una base general en todos los ámbitos temáticos, mientras que el AIFDD presenta conceptos armonizados del grupo de trabajo AIHWG Aeronautical Information Harmonization Working Group, conformado por miembros del DGIWG, contrapartes de la agencia civil, EUROCONTROL y OACI ${ }^{1}$.

\section{Estructura y objetos}

El catálogo AIFDD propone 102 objetos aeronáuticos clasificados en cinco subcategorías: a) aeródromos, movimiento de superficies y/o iluminación (Aerodromes, Movement Surfaces and/or Lighting), b) espacio aéreo y/o rutas (Airspace and/or Routes), c) ayudas de navegación, ayudas de aterrizaje, puntos y/o obstáculos (NAVAIDS, Landing Aids, Points and/or Obstacles), d) servicios, organizaciones y/o horarios (Services, Organisations and/or Timetables) y e) procedimientos de terminales (Terminal Procedures). Por su parte, el catálogo DFDD propone 137 objetos aeronáuticos clasificados en las mismas cinco subcategorías del AIFDD. Este es un cambio

\footnotetext{
${ }^{1}$ https://www.dgiwg.org/FAD/registers.jsp?register=DFDD
} 
Yépez Campoverde, J., Jiménez Calderón, L., Vázquez Hoehne, A. (2018): "Propuesta de catálogo normalizado de objetos aeronáuticos para el Ecuador”, GeoFocus (Artículos), $n^{\circ}$ 22, p. 21-42. ISSN: 1578-5157 http://dx.doi.org/10.21138/GF.479

importante en relación al antiguo FACC que incluía 49 objetos considerados únicamente en dos subcategorías: Rutas Aéreas (21 objetos) y Aeródromos (28 objetos). En la tabla 1 se puede observar un listado de los 26 objetos aeronáuticos que han prevalecido a través del tiempo, desde el FACC al AIFDD y DFDD respectivamente.

Tabla 1. Objetos aeronáuticos comunes entre FACC/AIFDD/DFDD

\begin{tabular}{|l|l|}
\hline CÓDIGO & \multicolumn{1}{|c|}{ NOMBRE } \\
Subcategoría: Aeródromos \\
\hline GA005 & Airspace_ \\
\hline GA010 & Air Traffic Service (ATS) Route Segment \\
\hline GA045 & Enroute Air Traffic Service (ATS) Route \\
\hline GA049 & Instrument Approach Procedure (IAP) \\
\hline GA055 & Way-point \\
\hline GA060 & Holding Procedure \\
\hline Subcategoria: Rutas Aéreas \\
\hline GB005 & Land Aerodrome \\
\hline GB007 & Aerodrome Boundary \\
\hline GB015 & Apron \\
\hline GB020 & Arresting System \\
\hline GB025 & Blast Barrier \\
\hline GB030 & Helipad \\
\hline GB035 & Heliport \\
\hline GB045 & Stopway \\
\hline GB047 & Aerodrome Reference Point (ARP) \\
\hline GB050 & Defensive Revetment \\
\hline GB055 & Runway \\
\hline GB056 & Runway Endpoint \\
\hline GB057 & Movement Area Shoulder \\
\hline GB058 & Point Abeam \\
\hline GB060 & Runway Radar Reflector \\
\hline GB065 & Water Aerodrome \\
\hline GB075 & Taxiway \\
\hline GB080 & Wind Direction Indicator \\
\hline GB090 & Runway Threshold \\
\hline GB220 & Aeronautical Obstacle \\
\hline & \\
\hline
\end{tabular}

Tanto el AIFDD como el DFDD, dejan entrever en su estructura su nueva codificación, sin embargo conservan también el código anterior referido al FACC con el fin de guardar correspondencia y facilitar al usuario la búsqueda de objetos geográficos. Para poder observar la clasificación de objetos aeronáuticos del AIFDD y DFDD según la codificación anterior referida a las dos subcategorías consideradas en el FACC (Rutas Aéreas y Aeródromos), se presenta la figura 3. Allí se observa que de los 102 objetos del AIFDD, 47 se ubican en Rutas Aéreas y 55 en Aeródromos, mientras que de los 137 objetos del DFDD, 68 se ubican en Rutas Aéreas y 69 en Aeródromos. 
Yépez Campoverde, J., Jiménez Calderón, L., Vázquez Hoehne, A. (2018): "Propuesta de catálogo normalizado de objetos aeronáuticos para el Ecuador”, GeoFocus (Artículos), $n^{\circ}$ 22, p. 21-42. ISSN: 1578-5157 http://dx.doi.org/10.21138/GF.479

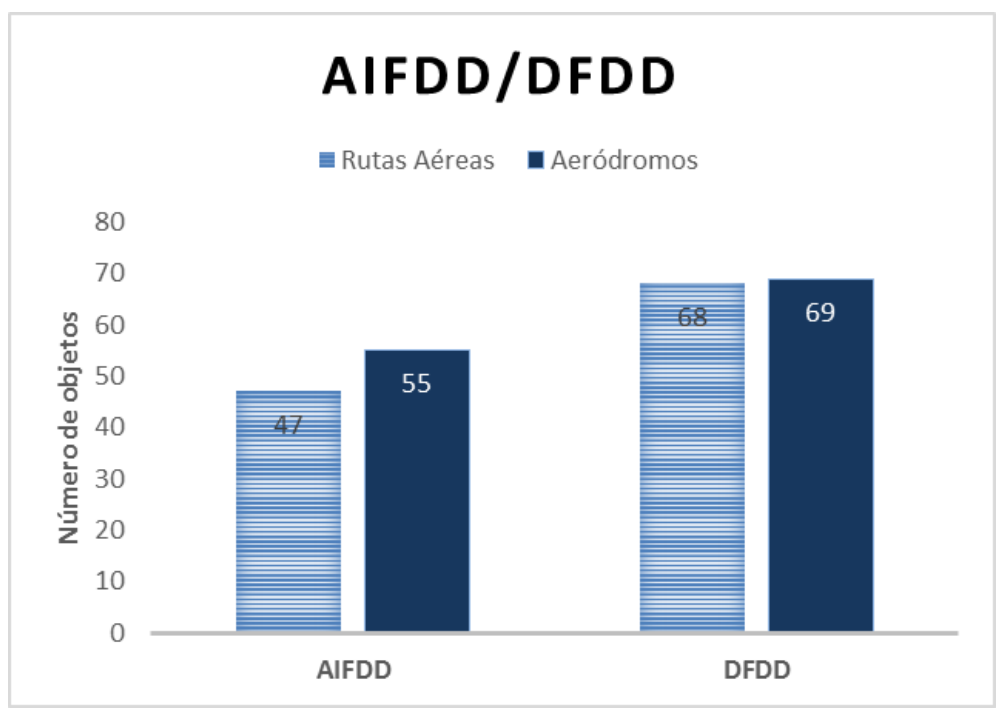

Figura 3. Relación de objetos aeronáuticos AIFDD y DFDD, según subcategorías antiguo FACC

Desde un análisis de similitud entre los tipos de objeto de los dos catálogos AIFDD y DFDD, se observan 101 objetos coincidentes. En general, los objetos de los dos catálogos conservan el mismo patrón de codificación anterior (FACC), sin embargo, se observa una diferencia en la clasificación, mientras que el AIFDD clasifica los objetos única y exclusivamente en las cinco subcategorías de la categoría Aeronáutica, el DFDD clasifica a estos objetos también en otras categorías y subcategorías fuera del ámbito aeronáutico, resultando que un objeto puede pertenecer a una subcategoría del campo aeronáutico y también pertenecer a otro campo, de forma duplicada. El $22 \%$ de los objetos del DFDD presentan una clasificación múltiple. En la figura 4 y figura 5 se observa la clasificación de objetos según cada catálogo respectivamente, notar que en la figura 5 se presenta de color gris el valor porcentual de los objetos clasificados de forma múltiple.

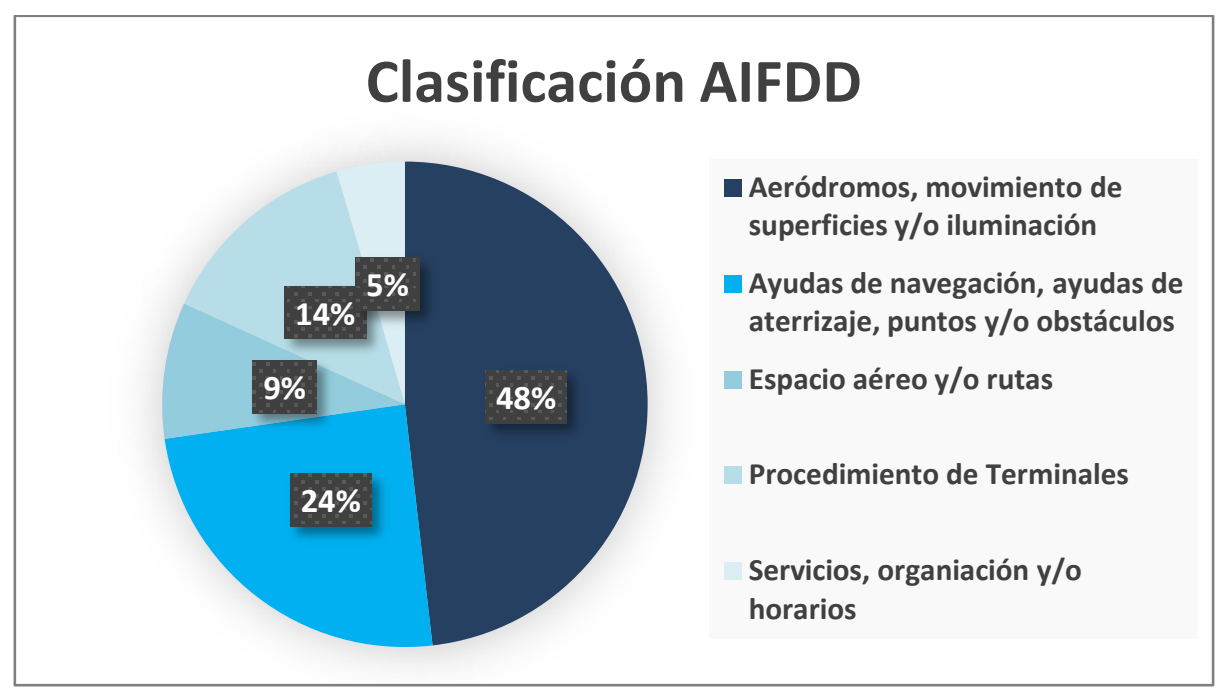

Figura 4. Clasificación de objetos aeronáuticos AIFDD. 
Yépez Campoverde, J., Jiménez Calderón, L., Vázquez Hoehne, A. (2018): "Propuesta de catálogo normalizado de objetos aeronáuticos para el Ecuador”, GeoFocus (Artículos), $n^{\circ} \quad 22, \quad p . \quad 21-42 . \quad$ ISSN: $1578-5157$ http://dx.doi.org/10.21138/GF.479

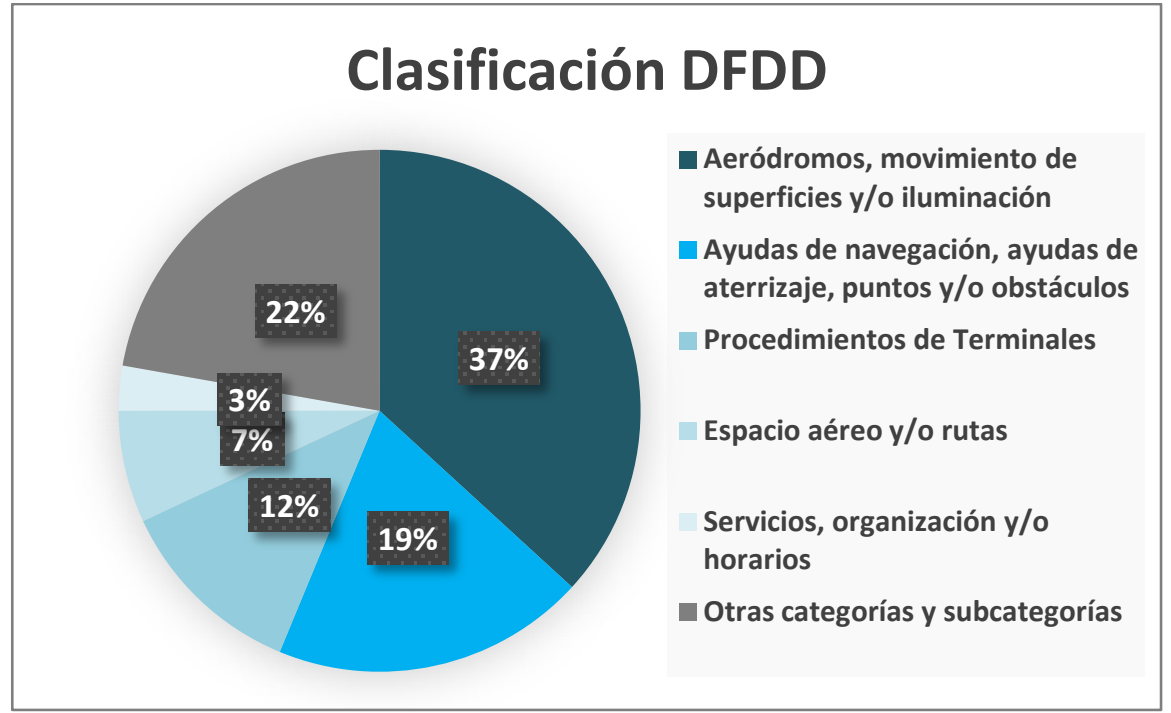

Figura 5. Clasificación de objetos aeronáuticos DFDD

\section{Semántica}

El nombre de los tipos de objeto ha ido variando de manera importante desde el FACC hasta el AIFDD y DFDD, sin embargo la coincidencia entre estos dos últimos es mayoritaria. El $31 \%$ de los nombres de los objetos se ha mantenido en el paso de la última versión FACC al AIFDD, mientras que el $69 \%$ restante ha cambiado. Se hace notar que, de modo general, aun cuando el nombre presente diferencias, se usan nombres similares que expresan la misma naturaleza del objeto.

En cuanto a las definiciones, los objetos del AIFDD y DFDD coinciden plenamente. Sin embargo, en vista de que el AIFDD presenta conceptos armonizados por el grupo de trabajo AIHWG, conformado, entre otros miembros, por la OACI, se realizó una comparación semántica entre las definiciones de los objetos del catálogo AIFDD con respecto a las definiciones presentes en el Anexo 4 de la OACI "Cartas Aeronáuticas" que contempla una serie de objetos aeronáuticos representados en la cartografía. Como resultado se observa que en gran parte no existe correspondencia entre las definiciones de los objetos aeronáuticos del catálogo y las definiciones de esos objetos descritas en este anexo OACI.

\subsection{Reunión de expertos}

Desde otro enfoque de análisis, se observa que los objetos del AIFDD solamente se encuentran asignados a las subcategorías que conforman la categoría Aeronáutica, mientras el DFDD propone a estos objetos dentro de subcategorías fuera del ámbito aeronáutico. Esto se debe a que los objetos aeronáuticos considerados en el AIFDD responden al interés estrictamente aeronáutico, mientras que los mismos objetos en el DFDD pueden interactuar con otros ámbitos no necesariamente aeronáuticos y por tanto, pueden adaptarse a diferentes enfoques y objetivos. Una 


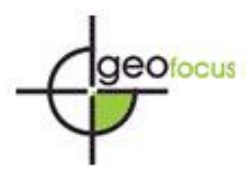

Revista Internacional de Ciencia y Tecnología de la Información Geográfica

International Review of Geographical Information Science and Technology

Yépez Campoverde, J., Jiménez Calderón, L., Vázquez Hoehne, A. (2018): "Propuesta de catálogo normalizado de objetos aeronáuticos para el Ecuador”, GeoFocus (Artículos), $n^{\circ}$ 22, p. 21-42. ISSN: 1578-5157 http://dx.doi.org/10.21138/GF.479

particularidad que presentan los dos catálogos es la posibilidad de que un tipo de objeto se encuentre dentro de varias subcategorías de forma simultánea. En el caso del AIFDD el tipo de objeto puede estar asignado a una o dos subcategorías, pero únicamente dentro del campo aeronáutico y en un mínimo número de tipos de objetos, mientras que en el DFDD el tipo de objeto puede estar asignado a una, dos o más subcategorías en un número considerable de objetos, inclusive fuera del ámbito aeronáutico, como ya se dijo anteriormente (figura 5). Esto no indica que el AIFDD y DFDD no cumplan con las normas ISO 19110 y 19135, en lo referente a un código único, sino que, posibilitan categorizar a un tipo de objeto desde distintas perspectivas, brindando al usuario la posibilidad de seleccionar la subcategoría o categoría más adecuada para un determinado fin.

Considerando la variedad de posibilidades y con el fin de asignar a cada tipo de objeto una única subcategoría, categoría, código y en consecuencia guardar correspondencia con lo expresado en las normas ISO 19119 y 19135, se procedió a realizar una reunión de expertos, con la participación de 7 pilotos de entre 48 y 65 años, con experiencia en información aeronáutica dentro del ámbito civil y militar, y con más de 25.000 horas de vuelo en su haber. La reunión de expertos comprendió una parte inicial de revisión de conceptos y un análisis marco, con énfasis en la importancia de un catálogo de objetos nacional. Se discutieron las diferencias más relevantes entre el catálogo AIFDD y DFDD, como resultado y en vista de que el enfoque del AIFDD está orientado de forma específica al campo aeronáutico, se seleccionó la estructura propuesta por este catálogo para ser sometida a discusión por los expertos. De esta forma se analizó la ubicación de cada tipo de objeto en una única subcategoría. Como consecuencia muchos de los objetos fueron ratificados según la clasificación del AIFDD, mientras que en otros casos, al no observar una correspondencia clara entre el objeto y la subcategoría, el grupo de expertos consideró asignar al tipo de objeto dentro de otra de las cinco subcategorías disponibles. Finalmente, esta discusión condujo necesariamente al análisis de la aplicabilidad de este catálogo en el ámbito nacional ecuatoriano.

\section{Descripción y análisis de resultados}

\subsection{Selección de tipos de objetos}

La selección de objetos no fue una tarea complicada ya que se tomaron como base los tipos de objetos comunes entre el catálogo DFDD y el catálogo AIFDD, que prácticamente son todos los comprendidos en el AIFDD, excepto uno. Se siguió este procedimiento, en vista de que por una parte es el DFDD quien de manera oficial reemplaza al estándar FACC según la DGIWG, pero por otra parte, los objetos del AIFDD son el resultado del consenso de varios actores, entre ellos organizaciones rectoras de la información aeronáutica a nivel internacional. Los objetos coincidentes tienen un enfoque más genérico y por tanto, se constituyen en un importante punto de partida para el Ecuador, que aún no tiene normativas, ni sistemas propios de producción de cartografía aeronáutica implementados. Sin embargo, la clasificación de los tipos de objetos en las subcategorías no fue una tarea sencilla, por una parte el DFDD clasificó los tipos de objetos indistintamente de la categoría Aeronáutica, por lo que esta clasificación fue totalmente desestimada, y por otra, la clasificación del AIFDD no correspondía totalmente con las actividades aeronáuticas que se desarrollan en el país. La estructura del catálogo y en tal virtud, la clasificación de los tipos de objetos en las subcategorías es relevante a la hora de la implementación del catálogo 


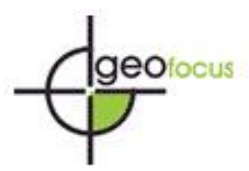

Yépez Campoverde, J., Jiménez Calderón, L., Vázquez Hoehne, A. (2018): "Propuesta de catálogo normalizado de objetos aeronáuticos para el Ecuador”, GeoFocus (Artículos), $n^{\circ}$ 22, p. 21-42. ISSN: $1578-5157$ http://dx.doi.org/10.21138/GF.479

como una posible normativa que orienta, condiciona y resuelve aspectos del ámbito aeronáutico en materia de cartografía, que a su vez es esencial en la seguridad de las actividades aéreas. Por lo expuesto, se consideró relevante la participación de expertos que permitieron clasificar y renombrar los tipos de objetos dentro de las cinco subcategorías del AIFDD y DFDD, anteriormente descritas.

Considerando los inconvenientes semánticos, se incluyeron para los tipos de objetos seleccionados las definiciones presentes en el anexo 4 de la OACI y, para los demás tipos no presentes en este anexo, se mantuvo la definición descrita en el AIFDD. La categoría Información Aeronáutica que en el FACC correspondía a (G), en el AIFDD y DFDD corresponde a (H) (véase tabla 2):

Tabla 2. Subcategorías del catálogo de tipos de objetos aeronáuticos

\begin{tabular}{|c|l|c|}
\hline Categoría & Subcategorías & Código \\
\hline \multirow{3}{*}{ Aeronáutica H } & Aeródromos, movimiento de superficies, iluminación & HA \\
\hline & Espacio Aéreo y/o Rutas & HB \\
\hline & $\begin{array}{l}\text { Ayudas de navegación, ayudas de aterrizaje, puntos y/o } \\
\text { obstáculos }\end{array}$ & HC \\
\cline { 2 - 3 } & \begin{tabular}{l} 
Servicios, organizaciones y/o horarios \\
\cline { 2 - 3 }
\end{tabular} & Procedimientos de terminales \\
\hline
\end{tabular}

\subsection{Asignación de tipos de objetos en subcategorías}

No se observa una marcada variación entre el AIFDD y el DFDD en cuanto al nombre y definición del tipo de objeto, por lo que el primer análisis con la ayuda de expertos se orientó a definir una única subcategoría para cada tipo de objeto, aquella con mayor correspondencia según los expertos. Siempre se seleccionó la decisión mayoritaria y no se presentaron casos de extrema discordancia. De este análisis se obtuvo como resultado que el $58 \%$ de los tipos de objetos asignados a una u otra subcategoría concuerda con el punto de vista de los expertos, mientras que el $44 \%$ de tipos de objetos, fueron reubicados en otras subcategorías para encontrar correspondencia con la realidad aeronáutica nacional ecuatoriana. Esta clasificación y el listado total de objetos aeronáuticos se pueden observar en el Anexo 1. De color verde se muestran los tipos de objetos clasificados en las subcategorías consideradas por el estándar AIFDD y a su vez por los expertos de forma coincidente. En color rojo se muestran los tipos de objetos clasificados según la propuesta de los expertos participantes que presenta discrepancia con la clasificación del AIFDD (ver apéndice 1). "Listado de tipos de objetos aeronáuticos del catálogo propuesto para el Ecuador". En la figura siguiente se puede observar que el DFDD clasifica al tipo de objeto indicador de dirección de aterrizaje (landing direction indicator) en la categoría Geografía Socio - Económica, mientras que el AIFDD coloca a este tipo de objeto en la categoría Aeronáutica. 
Yépez Campoverde, J., Jiménez Calderón, L., Vázquez Hoehne, A. (2018): "Propuesta de catálogo normalizado de objetos aeronáuticos para el Ecuador”, GeoFocus (Artículos), $n^{\circ}$ 22, p. 21-42. ISSN: 1578-5157 http://dx.doi.org/10.21138/GF.479

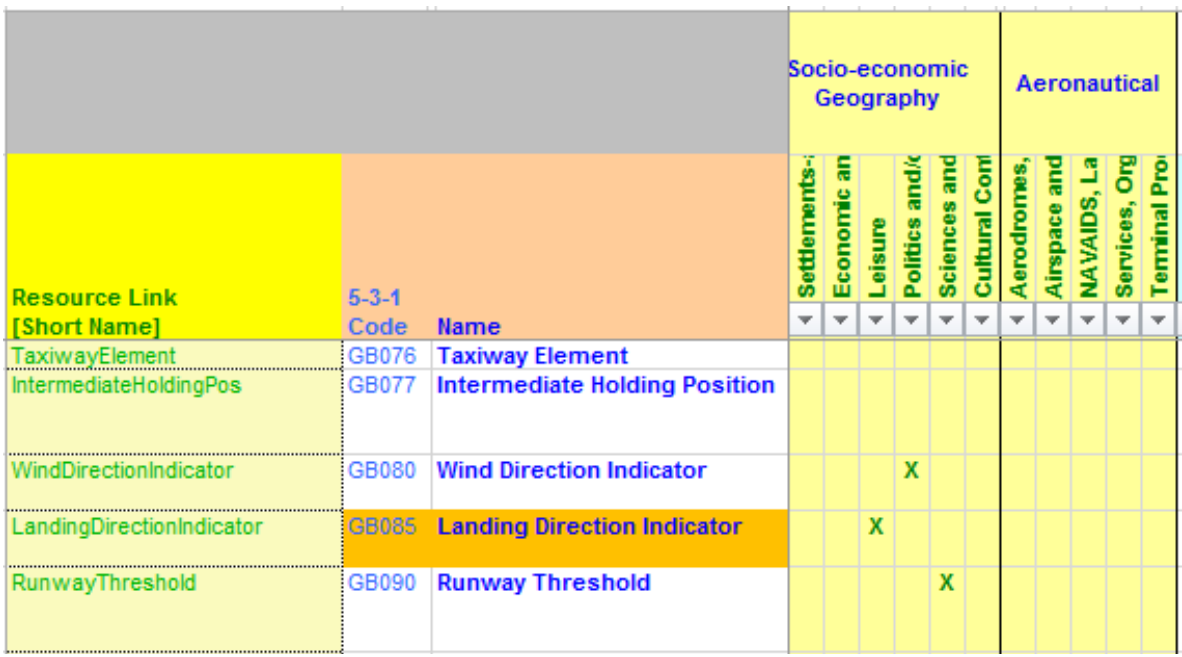

(a)

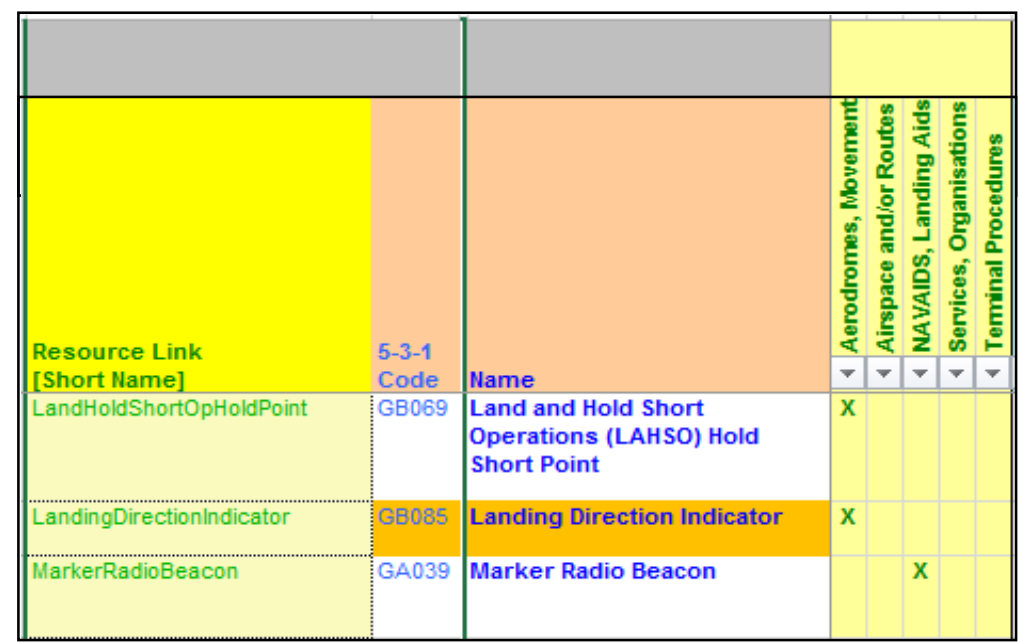

(b)

Figura 6. (a) Clasificación según DFDD. (b) Clasificación según AIFDD.

\subsection{Estructura y Diseño del Catálogo}

Para representar la estructura de los objetos y atributos, se diseñó una plantilla (véase tabla 3,4 y 5) que permite organizar los tipos de objetos aeronáuticos, sus principales características y elementos, con el fin de brindar al usuario mayor facilidad de uso. Esta plantilla contiene todos los campos obligatorios especificados, así como varios campos condicionales u opcionales para cada objeto y atributos de tipo texto, especificados en la norma ISO 19110. Los atributos con valores predefinidos (dominios) se han organizado de manera independiente, con el fin de mejorar la organización y para que puedan ser compartidos por varios objetos. La mayor parte de los atributos 
Yépez Campoverde, J., Jiménez Calderón, L., Vázquez Hoehne, A. (2018): "Propuesta de catálogo normalizado de objetos aeronáuticos para el Ecuador”, GeoFocus (Artículos), $n^{\circ}$ 22, p. 21-42. ISSN: 1578-5157 http://dx.doi.org/10.21138/GF.479

del AIFDD y DFDD son coincidentes, por lo tanto para su incorporación en el catálogo, se han tomado los atributos especificados por el estándar AIFDD traducidos al español.

En el ejemplo del tipo de objeto "Helipuerto" presentado en la plantilla (véase tabla 3), el código correspondiente según el estándar FACC sería GB035, según el AIFDD y el DFDD el código sería HA035. En este caso los expertos coinciden en ubicar el tipo de objeto en la categoría H y subcategoría A, sin embargo el código del tipo de objeto cambia de 035 a 660 , resultando finalmente HA660, donde H representa la categoría "Aeronáutica", A representa la subcategoría "Aeródromos" y 660 la numeración del objeto.

Tabla 3. Plantilla para caracterización de tipos de objetos aeronáuticos

\begin{tabular}{|c|c|c|c|c|c|}
\hline \multirow{8}{*}{ HА660 } & \multirow{2}{*}{$\frac{\text { Objeto: }}{\text { ódigo anterior }}$} & \multicolumn{4}{|l|}{ HELIPUERTO } \\
\hline & & GB035 & & & \\
\hline & \multirow{6}{*}{ Descripción: } & \multirow{6}{*}{$\begin{array}{l}\text { Aeródromo o área definida sobre } \\
\text { una estructura artificial destinada } \\
\text { a ser utilizada, total o parcialmente, } \\
\text { para la llegada, la salida o el } \\
\text { movimiento de superficie de los } \\
\text { helicópteros (OACI - Anexo } 4 \text {, } \\
\text { 2009). }\end{array}$} & \multicolumn{2}{|r|}{ Categoría } & AERONAÚTICA \\
\hline & & & \multicolumn{2}{|c|}{ Subcategoría: } & TERMINAL PROCEDURES \\
\hline & & & \multicolumn{2}{|r|}{ Geometría: } & Punto / Polígono \\
\hline & & & \multicolumn{2}{|c|}{ Feature Dataset: } & H_TERMINAL_PROCEDURES \\
\hline & & & \multirow{2}{*}{\multicolumn{2}{|c|}{ Feature class }} & HELIPUERTO_P \\
\hline & & & & & HELIPUERTO_A \\
\hline \multicolumn{5}{|c|}{ Atributos } & Dominios \\
\hline Código & Nombre & Descripción & $\begin{array}{l}\text { Tipo de } \\
\text { dato }\end{array}$ & Extensión & Valores del dominio \\
\hline fcode & Código & $\begin{array}{l}\text { Código de identificación del objeto } \\
\text { geográfico dentro del Catálogo }\end{array}$ & text & 5 & HE035 \\
\hline desc & Descripción & $\begin{array}{l}\text { Descripción del objeto en lenguaje } \\
\text { natural }\end{array}$ & text & 250 & Texto libre \\
\hline txt & Observaciones & Texto aclaratorio del objeto & text & 250 & Texto libre \\
\hline nam & Nombre oficial & Nombre oficial del objeto & text & 80 & Texto libre \\
\hline iko & $\begin{array}{l}\text { Identificador de } \\
\text { localización }\end{array}$ & $\begin{array}{l}\text { Identificador de localización } \\
\text { designado por la OACI }\end{array}$ & text & 5 & Texto libre \\
\hline esn & Elevación & Elevación sobre el nivel del mar & double & & Texto libre \\
\hline pod & $\begin{array}{l}\text { Operaciones } \\
\text { diarias }\end{array}$ & Promedio de operaciones diarias & double & & Texto libre \\
\hline csi & $\begin{array}{l}\text { Categoría de } \\
\text { servicio }\end{array}$ & Categoría de servicio SEI & \multicolumn{3}{|c|}{ Ver dominios asociados } \\
\hline acc & $\begin{array}{l}\text { Categoría de } \\
\text { precisión }\end{array}$ & $\begin{array}{l}\text { Descripción de la posición } \\
\text { geográfica }\end{array}$ & \multicolumn{3}{|c|}{ Ver dominios asociados } \\
\hline use & Categoría de uso & Categoría del uso del objeto & \multicolumn{3}{|c|}{ Ver dominios asociados } \\
\hline
\end{tabular}

Cada tipo de objeto tiene asignado un código único y por tanto, una única categoría y subcategoría, además de su definición en español, que ha sido tomada del Anexo 4 de la OACI en aquellos casos en los que existe la definición del tipo de objeto, caso contrario se ha tomado la definición del estándar AIFDD. También se especifica la organización en la base de datos geográfica a través del Feature Dataset y el, o los Feature Class, cuyos nombres también se han 
Yépez Campoverde, J., Jiménez Calderón, L., Vázquez Hoehne, A. (2018): "Propuesta de catálogo normalizado de objetos aeronáuticos para el Ecuador”, GeoFocus (Artículos), $n^{\circ}$ 22, p. 21-42. ISSN: $1578-5157$ http://dx.doi.org/10.21138/GF.479

estandarizado en función de la categoría y de la geometría del tipo de objeto. Aun cuando no es competencia de un catálogo de acuerdo a los estándares, la definición de la geometría del tipo de objeto, para fines prácticos, en este catálogo se ha definido, las posibles geometrías que puede tomar el objeto para su representación. En el caso de una línea se coloca el nombre del objeto seguido de un guion bajo y la letra mayúscula L, en el caso de un área la letra A, y en el caso de un punto la letra $\mathrm{P}$.

La vinculación de la geometría a los tipos de objetos presenta varias ventajas en el uso de la cartografía aeronáutica. Por una parte, permite que el usuario tenga un indicador adicional de aproximación de la aeronave al tipo de objeto. Si tomamos el tipo de objeto pista, considerando que una aeronave se encuentra a una distancia considerable, éste tipo de objeto se desplegará como línea con el nombre PISTA_L, mientras que si la aeronave se encuentra en las aproximaciones de este tipo de objeto, se desplegará como polígono con el nombre PISTA_A. Para el tipo de objetos obstáculos en las operaciones aéreas, este indicador de aproximación podría cobrar mayor importancia.

Desde otro punto de vista, y debido a que se han observado diferencias en la representación de tipos de objetos aeronáuticos en los distintos continentes (Moya 2016), la inclusión de aspectos geométricos en el catálogo, permite diseñar una carta aeronáutica de acuerdo a lo establecido al anexo 4 "cartas aeronáuticas de la OACI, logrando que conforme a la escala, se utilicen los elementos y su representación más adecuada, facilitando la eficiencia de las cartas aeronáuticas y alcanzando la usabilidad deseada, logrando que exista coherencia técnica y semántica, tanto gráfica como alfanumérica, es decir, en la representación cartográfica y en la estructura de las bases de datos. Es importante considerar además que según expertos como Moya (2016), una mayor usabilidad tiene un impacto positivo en la seguridad de las operaciones aéreas.

Tabla 4. Caracterización de atributos

\begin{tabular}{|c|l|l|c|c|}
\hline LIS & \multicolumn{4}{c|}{ VARIABILIDAD DEL SISTEMA DE INTENSIDAD DE LUZ } \\
\hline Código & Nombripción: el nivel de intensidad de luz & $\begin{array}{c}\text { Tipo de } \\
\text { dato }\end{array}$ & Extensión \\
\hline LIS & Código & $\begin{array}{l}\text { Código alfanumérico para cada nivel de } \\
\text { intensidad de luz }\end{array}$ & $\begin{array}{c}\text { Short } \\
\text { integer }\end{array}$ & \\
\hline LIS_ETIQ & Etiqueta & $\begin{array}{l}\text { Etiqueta que identifica cada nivel de } \\
\text { intensidad de luz }\end{array}$ & text & 80 \\
\hline LiS_DESC & Descripción & $\begin{array}{l}\text { Describe en lenguaje natural el nivel de } \\
\text { intensidad de luz y las implicaciones de } \\
\text { ser el caso }\end{array}$ & text & 250 \\
\hline
\end{tabular}


Yépez Campoverde, J., Jiménez Calderón, L., Vázquez Hoehne, A. (2018): "Propuesta de catálogo normalizado de objetos aeronáuticos para el Ecuador”, GeoFocus (Artículos), $n^{\circ}$ 22, p. 21-42. ISSN: 1578-5157 http://dx.doi.org/10.21138/GF.479

Tabla 5. Ejemplo de caracterización de los dominios de un atributo

\begin{tabular}{|c|c|c|}
\hline $\begin{array}{l}\text { Código del } \\
\text { dominio }\end{array}$ & $\begin{array}{l}\text { Etiqueta del } \\
\text { dominio }\end{array}$ & Descripción del dominio \\
\hline LIS 0 & DESCONOCIDO & $\begin{array}{l}\text { INDICA QUE UN VALOR DE ATRIBUTO NO } \\
\text { ESTÁ DEFINIDO O NO SE CONOCE LA } \\
\text { CARACTERÍSTICA DEL OBJETO }\end{array}$ \\
\hline LIS 1 & ALTO & $\begin{array}{l}\text { INTENSIDAD DE LUZ ALTA, EN ESTE } \\
\text { CASO, UN CONTROL DE INTENSIDAD } \\
\text { ADECUADO SERÁ INCORPORADO PARA } \\
\text { PERMITIR EL AJUSTE DE LA INTENSIDAD } \\
\text { DE LUZ PARA CUMPLIR CON LAS } \\
\text { CONDICIONES PREESTABLECIDAS }\end{array}$ \\
\hline LIS 2 & MEDIO & INTENSIDAD DE LUZ MEDIA \\
\hline LIS 3 & BAJO & INTENSIDAD DE LUZ BAJA \\
\hline LIS 998 & NO APLICABLE & $\begin{array}{l}\text { INDICA QUE UN VALOR DE ATRIBUTO NO } \\
\text { ES APLICABLE }\end{array}$ \\
\hline
\end{tabular}

En el extinto FACC los valores de los atributos predefinidos, expresados en listas controladas, podían considerar una serie de valores, entre ellos el valor 0 para "desconocido" (LIS 0 para el ejemplo de la tabla 5), un valor 998 para "no aplicable" y un valor 999 para "otro". A diferencia del FACC, el AIFDD y DFDD no consideran explícitamente los valores 0, 998 y LIS 999. Por otra parte, considerando que estos valores suelen utilizarse con cierta ligereza para almacenar cualquier tipo de información, lo cual genera serios inconvenientes en las bases de datos, se ha considerado adecuado excluir en el catálogo propuesto los valores 999, sin embargo se ha creído conveniente conserva el valor 0 "desconocido" a efectos prácticos de no dejar registros vacíos por la no existencia de información en un determinado caso y el valor 998, ya que en efecto podrían haber este tipo de casos.

\section{Conclusiones}

Dada la importancia de la información aeronáutica para la seguridad de las operaciones aéreas, es necesario definir entre otros estándares, un catálogo de tipos de objetos aeronáuticos normalizado, para poder gestionar de forma eficiente los servicios de información aeronáutica. A través de normas internacionales como las que propone ISO TC211, se asegura la comprensión por parte de los países miembros y de esa forma, se logra la interoperabilidad en un ámbito como el aeronáutico, que claramente requiere la colaboración de varios estados. En tal sentido, el catálogo de objetos propuesto busca facilitar la comprensión de la información aeronáutica a nivel local, a través del diseño y la estructura planteados. Facilita también su trasposición a una base de datos geográfica para el almacenamiento de la información, estandarizando la denominación de los datos y conjuntos de datos. Por otra parte, al seguir los lineamientos de las normas ISO 19110, 19126 y 19135 en lo relativo a los requisitos de un catálogo de objetos, a la codificación y los registros, posibilita un uso que sobrepasa el ámbito nacional. 


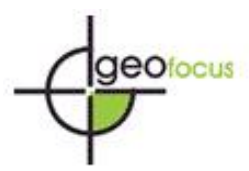

Yépez Campoverde, J., Jiménez Calderón, L., Vázquez Hoehne, A. (2018): "Propuesta de catálogo normalizado de objetos aeronáuticos para el Ecuador”, GeoFocus (Artículos), $n^{\circ}$ 22, p. 21-42. ISSN: 1578-5157 http://dx.doi.org/10.21138/GF.479

El AIFDD representa una buena propuesta de catálogo temático, en razón de que representa el consenso de varios actores involucrados con información aeronáutica, vinculados a través del grupo de trabajo AIHWG de la DGIWG, lo cual no se observa en otros ámbitos temáticos. Sin embargo, no se evidencia una total correspondencia sobre las actividades aeronáuticas del Ecuador que involucran tipos de objetos geográficos en este campo. Un ejemplo se presenta en la figura 7 que se presenta a continuación. Ahí se observa que según la clasificación del AIFDD, el tipo de objeto GA021 Área de Altitud Segura (Safe Altitude Area) pertenece a la subcategoría Procedimientos de Terminal (Terminal Procedures), sin embargo los expertos consultados consideraron que éste tipo de objeto debía pertenecer a la subcategoría relacionada con obstáculos (NAVAIDS, Landing Aids, Points and/or Obstacles).

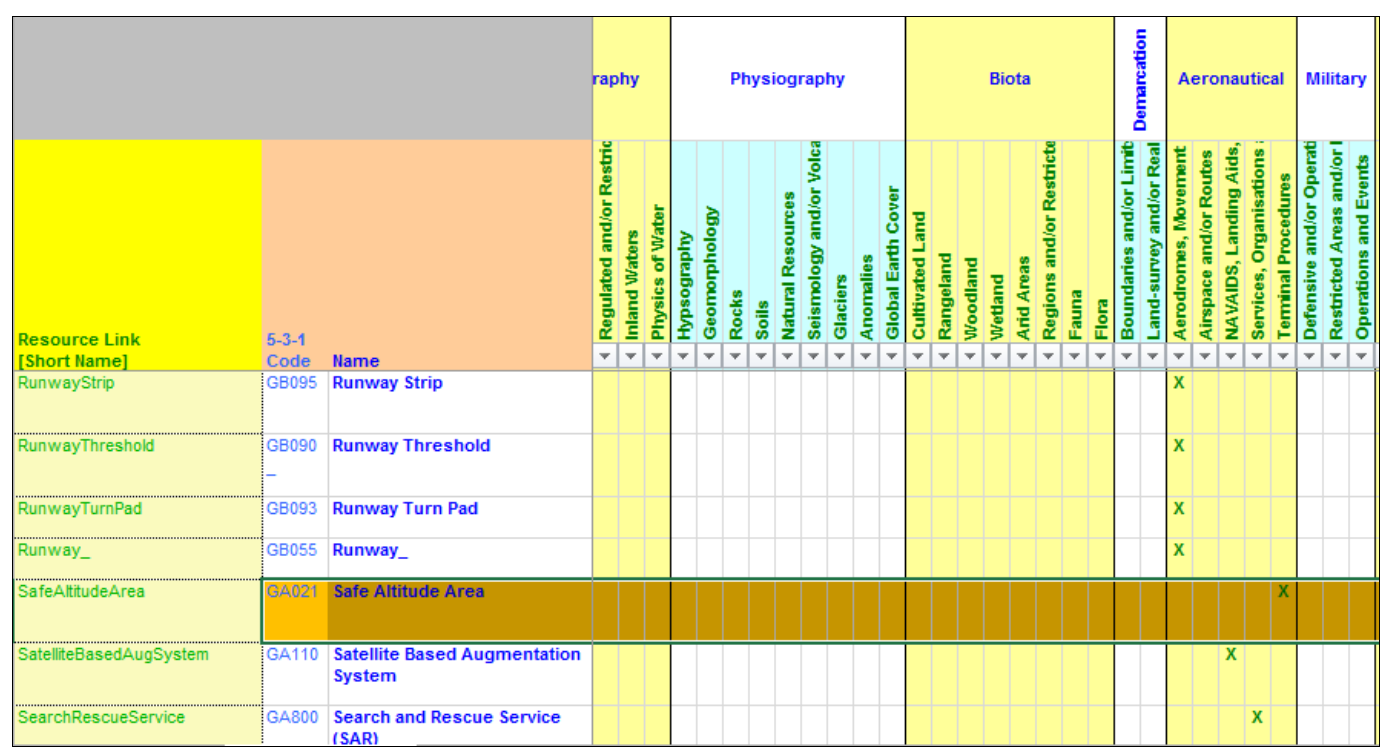

Figura 7. Clasificación del tipo de objeto GA021 según AIFDD.

Por lo expuesto, la participación de expertos a nivel nacional posibilita alcanzar una determinada aplicabilidad de este tipo de estándares internacionales en una región o país en particular. Este catálogo es un punto de partida para futuras discusiones donde participen más actores vinculados a las actividades aeronáuticas, considerando que para alcanzar la interoperabilidad semántica se requiere un trabajo continuo y mancomunado.

Existen varios catálogos presentados a la DGIWG por organizaciones como la Organización de Tratados del Atlántico Norte (OTAN), el Centro Satelital de la Unión Europea (EUSC), países como Dinamarca, Estados Unidos y Alemania en espera de aprobación total o parcial y por lo tanto no se consideran oficiales. En este sentido, existe la posibilidad de remitir una propuesta de catálogo nacional, así como realizar aportaciones específicas en los catálogos disponibles.

El principal aporte de este nuevo catálogo es la sinergia entre el trabajo realizado por el DGIWG a lo largo de estos últimos años, y la correspondencia semántica con las definiciones aeronáuticas especificadas por la OACI, en su Anexo 4 "Cartas Aeronáuticas" en su edición del año 


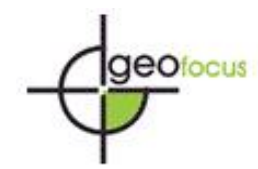

Yépez Campoverde, J., Jiménez Calderón, L., Vázquez. Hoehne, A. (2018): "Propuesta de catálogo normalizado de objetos aeronáuticos para el Ecuador”, GeoFocus (Artículos), $n^{\circ}$ 22, p. 21-42. ISSN: 1578-5157 http://dx.doi.org/10.21138/GF.479

2009. Del mismo modo, la revisión de expertos en este tema, garantiza la aplicabilidad de la propuesta del AIFDD para el Ecuador.

\section{Referencias bibliográficas}

Aguilar, E., Bautista, G. y Sánchez, E. (2009). Reporte Técnico Sistemas de Navegación Aérea, VOR y DME. Tesis Doctoral Instituto Politécnico Nacional de México, México D.F.

Ariza, F., Rodríguez, F. (2008). Introducción a la Normalización en Información Geográfica: La Familia ISO 19100, Grupo de Investigación en Ingeniería Cartográfica, Universidad de Jaén.

Bravo, M., Rodríguez, C., Valdés, M., Benavides, D., Criado, M. y Crespo, M. (2010). Experiencias en la catalogación de la información geográfica aeronáutica de AENA. Jornadas de Infraestructuras de Datos Espaciales JIIDE10.

Calvo, J. (2003). Fundamento de navegación aérea. Ediciones de la Universidad Autónoma de Madrid. ISSN/ISBN 84-7477-843-3.

EUROCONTROL y European Commision, (2010). SESAR Annual Report 2009. 28. Brussels: Single European Sky ATM Research Joint Undertaking (SESARJU).

EUROCONTROL y European Commision, (2011). SESAR Magazine 8. Brussels: Single European Sky ATM Research Joint Undertaking (SESARJU).

FAA, (2012). NextGEN Implementation Plan. 105. Washington, DC: Department of Transportation, Federal Aviation Administration (FAA).

ISO (2005). ISO 19110:2005. Geographic information -- Methodology for feature cataloguing. International organization for standardization.

ISO (2009). ISO 19126:2009. Geographic information -- Feature concept dictionaries and registers. International organization for standardization.

Latingeo, (2009). Resumen Norma ISO 19135. Laboratorio de Tecnologías de Información Geográfica Latingeo Catamarca. Argentina.

Mas, S., Rodríguez, A. y Bernabé, M. (2012). Compartir datos geográficos. En: Bernabé, M. y López, C., Fundamentos de las Infraestructuras de Datos Espaciales. Madrid, UPM-Press, Serie Científica. ISBN: 978-84-939196-6-5, pp. 31-40.

Moya, J., Benavides, D., Vaca, S., Siabato, W., Valdés, M. y Bernabé, M. (2010). La publicación de información aeronáutica: Hacia una nueva eAIP basada en geoservicios. I Jornadas Ibéricas de Infraestructuras de Datos Espaciales (JIIDE 2010), 27 - 29 de Oct 2010. Lisboa, Portugal.

Moya Honduvilla, J. (2016). Contribución a la mejora de la expresión gráfica de las cartas aeronáuticas (Doctoral dissertation, UPM - ETSI Topografía, Geodesia y Cartografía).

OACI, (2004). Annex 15 to the Convention on International Civil Aviation OrganizationAeronautical Information Services" / Twelfth Edition. (Pp. 3-1). Montreal, Canada.

OACI, (2001). Annex 11 to the Convention on International Civil Aviation Organization: Services Air Traffic Control / the thirteenth edition. Montreal, Canadá. 


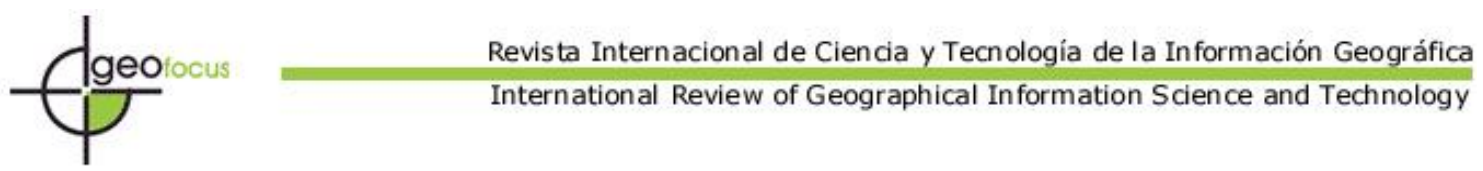

Yépez Campoverde, J., Jiménez Calderón, L., Vázquez Hoehne, A. (2018): "Propuesta de catálogo normalizado de objetos aeronáuticos para el Ecuador”, GeoFocus (Artículos), $n^{\circ}$ 22, p. 21-42. ISSN: 1578-5157 http://dx.doi.org/10.21138/GF.479

Pérez, L., Arnaldo, R., Sáez, F., Blanco J y Gómez V. (2013). Introducción al Sistema de Navegación Aérea. Escuela Técnica Superior de Ingeniería Aeronáutica y del Espacio (ETSIAE). Grupo editorial Gaceta.

Sáez, F. y Portillo, Y. (2003). Descubrir la navegación aérea, (1ra). Madrid: Aeropuertos Españoles y Navegación Aérea AENA.

STANAG (1998). STANAG 7074: Digital Geographic Information Exchange Standard (DIGEST). North Atlantic Treaty Organization Standardization Agreement. Brussels 
Yépez Campoverde, J., Jiménez Calderón, L., Vázquez Hoehne, A. (2018): "Propuesta de catálogo normalizado de objetos aeronáuticos para el Ecuador”, GeoFocus (Artículos), $n^{\circ}$ 22, p. 21-42. ISSN: 1578-5157 http://dx.doi.org/10.21138/GF.479

\section{APÉNDICE 1. Listado de tipos de objetos aeronáuticos propuestos para el Ecuador}

De color verde se destacan las concordancias de los expertos con el AIFDD, mientras que de color rojo, las discrepancias y por tanto, la propuesta de los expertos.

\begin{tabular}{|c|c|c|c|c|c|c|c|}
\hline & & & & & eronáutica & & \\
\hline $\begin{array}{l}\text { CÓDIGO } \\
\text { NUEVO }\end{array}$ & $\begin{array}{l}\text { CÓDIGO } \\
\text { ANTERIOR }\end{array}$ & NOMBRE & 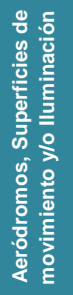 & 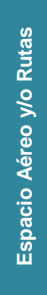 & 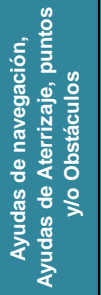 & 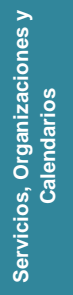 & 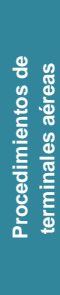 \\
\hline HB005 & GA005 & Espacio aéreo & & $\mathbf{X}$ & & & \\
\hline HA010 & GA010 & Servicio de tránsito aéreo ATS Segmento Ruta & $\mathbf{x}$ & & & & \\
\hline $\mathrm{HCO} 20$ & GA021 & Área de altitud segura & & & $\mathbf{x}$ & & \\
\hline $\mathrm{HCO} 23$ & GA023 & Altitud de terminales de llegada TAA & & & $\mathbf{x}$ & & \\
\hline $\mathrm{HCO} 24$ & GA024 & Sector de altitud terminal de Ilegada & & & $\mathbf{x}$ & & \\
\hline HCO35 & GA035 & Servicio de Radio navegación aeronáutica & & & $\mathbf{x}$ & & \\
\hline HA036 & GA036 & Baliza de radio omnidireccional VHF & $\mathbf{x}$ & & & & \\
\hline HA037 & GA037 & Baliza de ayuda táctica de radio navegación & $\mathbf{x}$ & & & & \\
\hline HA038 & GA038 & Baliza de radio no - direccional & $\mathbf{x}$ & & & & \\
\hline HA039 & GA039 & Marcador de radio baliza & $\mathbf{x}$ & & & & \\
\hline $\mathrm{HCO40}$ & GA040 & Sistema de navegación global por satélite GNSS & & & $\mathbf{x}$ & & \\
\hline HC041 & GA041 & Equipo radiotelemétrico & & & $\mathbf{x}$ & & \\
\hline $\mathrm{HCO} 043$ & GA043 & Limitación del servicio de radio navegación & & & $\mathbf{x}$ & & \\
\hline HB044 & GA044 & Cobertura del servicio de radio navegación & & $\mathbf{x}$ & & & \\
\hline HB045 & GA045 & Ruta ATS en ruta & & $\mathbf{x}$ & & & \\
\hline HB049 & GA049 & Procedimiento de aproximación instrumental & & $\mathbf{x}$ & & & \\
\hline HB055 & GA055 & Punto de ruta & & $\mathbf{x}$ & & & \\
\hline HB057 & GA057 & Punto significativo & & $\mathbf{x}$ & & & \\
\hline HB058 & GA058 & Punto designado & & $\mathbf{x}$ & & & \\
\hline HB059 & GA059 & Punto significativo del espacio aéreo & & $\mathbf{x}$ & & & \\
\hline HA060 & GA060 & Procedimiento de espera & $\mathbf{x}$ & & & & \\
\hline $\mathrm{HC} 090$ & GA090 & Sistema de aterrizaje instrumental & & & $\mathbf{x}$ & & \\
\hline HC091 & GA091 & Localizador del sistema de aterrizaje instrumental & & & $\mathbf{x}$ & & \\
\hline $\mathrm{HC} 092$ & GA092 & Sistema de aterrizaje por microondas & & & $\mathbf{x}$ & & \\
\hline HCO93 & GA093 & Azimut del sistema de aterrizaje por microondas & & & $\mathbf{x}$ & & \\
\hline HC094 & GA094 & Elevación del sistema de aterrizaje por microondas & & & $\mathrm{x}$ & & \\
\hline HA095 & GA095 & Radar de precisión de aproximación & $\mathbf{x}$ & & & & \\
\hline HCO96 & GA096 & Sistema de navegación especial & & & $\mathbf{x}$ & & \\
\hline HE097 & GA097 & Estación del sistema de navegación especial & & & & & $\mathbf{x}$ \\
\hline HE098 & GA098 & Trayectoria de planeo del sistema de aterrizaje instrumental & & & & & $\mathbf{x}$ \\
\hline HCO99 & GA099 & Brújula - localizador & & & $\mathbf{x}$ & & \\
\hline HC110 & GA110 & Sistema de aumento basado en satélite & & & $\mathbf{x}$ & & \\
\hline HD210 & GA210 & Segmento de procedimiento de vuelo & & & & $\mathbf{X}$ & \\
\hline HE220 & GA220 & Llegada instrumental estándar & & & & & $\mathrm{x}$ \\
\hline HD230 & GA230 & Despegue instrumental estándar & & & & & $\mathbf{x}$ \\
\hline HB250 & GA250 & Segmento de transición & & $\mathbf{x}$ & & & \\
\hline HB260 & GA260 & Segmento de aproximación inicial & & $\mathbf{x}$ & & & \\
\hline
\end{tabular}


Yépez Campoverde, J., Jiménez Calderón, L., Vázquez Hoehne, A. (2018): "Propuesta de catálogo normalizado de objetos aeronáuticos para el Ecuador”, GeoFocus (Artículos), $n^{\circ} \quad 22, \quad$. 21-42. ISSN: $1578-5157$ http://dx.doi.org/10.21138/GF.479

\begin{tabular}{|c|c|c|c|c|c|c|c|}
\hline & & & \multicolumn{5}{|c|}{ Aeronáutica } \\
\hline $\begin{array}{l}\text { CÓDIGO } \\
\text { NUEVO }\end{array}$ & $\begin{array}{c}\text { CÓDIGO } \\
\text { ANTERIOR }\end{array}$ & NOMBRE & 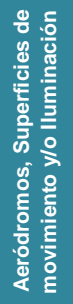 & 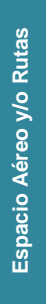 & 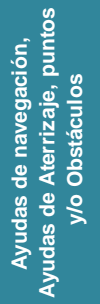 & 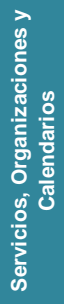 & 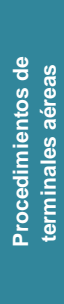 \\
\hline HB270 & GA270 & Segmento de aproximación intermedio & & $\mathrm{x}$ & & & \\
\hline HA280 & GA280 & Segmento de aproximación final & $\mathbf{x}$ & & & & \\
\hline HE290 & GA290 & Segmento de aproximación perdido & & & & & $\mathbf{x}$ \\
\hline HA500 & GA500 & Segmento de llegada & $x$ & & & & \\
\hline HA510 & GA510 & Segmento de despegue & $\mathbf{x}$ & & & & \\
\hline HA520 & GA520 & Procedimiento de reabastecimiento aéreo & $\mathbf{x}$ & & & & \\
\hline HA530 & GA530 & Pista de reabastecimiento aéreo & $\mathbf{x}$ & & & & \\
\hline HA540 & GA540 & Patrón de anclaje de reabastecimiento aéreo & $\mathbf{x}$ & & & & \\
\hline HB550 & GA550 & Punto de reabastecimiento aéreo & & $\mathrm{x}$ & & & \\
\hline HE800 & GA800 & Servicio de rescate y búsqueda & & & & & $\mathrm{x}$ \\
\hline HA550 & GB001 & Aeródromo & $\mathbf{x}$ & & & & \\
\hline HA560 & GB005 & Aeródromo en tierra & $\mathbf{x}$ & & & & \\
\hline HA570 & GB007 & Límites de aeródromo & $\mathbf{x}$ & & & & \\
\hline $\mathrm{HC} 120$ & GB008 & Marcas de aeródromo & & & $\mathbf{x}$ & & \\
\hline HA580 & GB011 & Área de iluminación de movimiento del aeródromo & $\mathbf{x}$ & & & & \\
\hline HD240 & GB012 & Planta de luz aeronáutica & & & & $\mathbf{x}$ & \\
\hline HA590 & GB013 & Baliza de aeródromo & $\mathbf{x}$ & & & & \\
\hline HA600 & GB015 & Plataforma (Apron) & $\mathbf{x}$ & & & & \\
\hline HA610 & GB016 & Parqueo de aeronaves & $\mathbf{x}$ & & & & \\
\hline HA620 & GB017 & Punto caliente del aeródromo & $\mathbf{x}$ & & & & \\
\hline HA630 & GB018 & Posición de parqueo de aviones aislada & $\mathbf{x}$ & & & & \\
\hline HA640 & GB020 & Sistema de detención & $\mathbf{x}$ & & & & \\
\hline HC130 & GB025 & Barrera de exploción & & & $\mathbf{x}$ & & \\
\hline HA650 & GB030 & Zona de helipuerto & $\mathbf{x}$ & & & & \\
\hline HE800 & GB032 & Aproximación final y área de despegue & & & & & $\mathbf{x}$ \\
\hline $\mathrm{HC} 140$ & GB033 & Contacto y zona de elevación & & & $\mathbf{x}$ & & \\
\hline HA660 & GB035 & Helipuerto & $\mathbf{x}$ & & & & \\
\hline HA670 & GB036 & Área de seguridad de aproximación final y despegue & $\mathbf{x}$ & & & & \\
\hline HA680 & GB045 & Zona de parada (stopway) & $\mathbf{x}$ & & & & \\
\hline $\mathrm{HC} 150$ & GB047 & Punto de referencia del aeródromo & & & $\mathbf{x}$ & & \\
\hline HA690 & GB048 & Área sensible del sistema de aterrizaje instrumental & $\mathbf{x}$ & & & & \\
\hline HA700 & GB049 & Área crítica del sistema de aterrizaje instrumental & $\mathbf{x}$ & & & & \\
\hline HE810 & GB050 & Servicio de revestimiento de aviones & & & & & $\mathrm{x}$ \\
\hline HE820 & GB052 & Dirección de la pista & & & & & $\mathbf{x}$ \\
\hline HA710 & GB055 & Pista & $\mathbf{x}$ & & & & \\
\hline HC160 & GB056 & Punto final de la pista & & & $\mathbf{x}$ & & \\
\hline HA720 & GB057 & Área de resalte & $\mathbf{x}$ & & & & \\
\hline HA730 & GB058 & Punto al través & $\mathbf{X}$ & & & & \\
\hline
\end{tabular}


Yépez Campoverde, J., Jiménez Calderón, L., Vázquez Hoehne, A. (2018): "Propuesta de catálogo normalizado de objetos aeronáuticos para el Ecuador”, GeoFocus (Artículos), $n^{\circ}$ 22, p. 21-42. ISSN: $1578-5157$ http://dx.doi.org/10.21138/GF.479

\begin{tabular}{|c|c|c|c|c|c|c|c|}
\hline \multirow[b]{2}{*}{$\begin{array}{l}\text { CÓDIGO } \\
\text { NUEVO }\end{array}$} & \multirow[b]{2}{*}{$\begin{array}{l}\text { CÓDIGO } \\
\text { ANTERIOR }\end{array}$} & \multirow[b]{2}{*}{ NOMBRE } & \multicolumn{5}{|c|}{ Aeronáutica } \\
\hline & & & 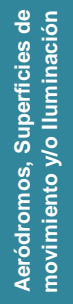 & 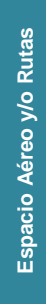 & 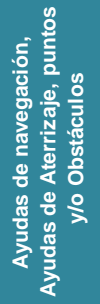 & 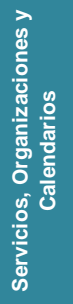 & 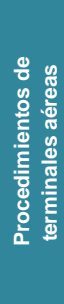 \\
\hline HC170 & GB060 & Reflector Radar de Pista & & & $\mathbf{x}$ & & \\
\hline HA740 & GB065 & Aeródromo acuático & $\mathbf{x}$ & & & & \\
\hline HE830 & GB069 & Operaciones a corto alcance LAHSO & & & & & $\mathbf{x}$ \\
\hline HA750 & GB075 & Calle de rodaje (taxiway) & $\mathbf{x}$ & & & & \\
\hline HA760 & GB077 & Posición intermedia de espera & $\mathbf{x}$ & & & & \\
\hline HA770 & GB080 & Indicador de dirección del viento & $\mathrm{x}$ & & & & \\
\hline HC180 & GB085 & Indicador de dirección de aterrizaje & & & $\mathbf{x}$ & & \\
\hline HE840 & GB090 & Umbral de pista & & & & & $\mathbf{x}$ \\
\hline HC190 & GB091 & Área extrema de seguridad de pista & & & $\mathbf{x}$ & & \\
\hline HE840 & GB092 & Posición en espera de pista & & & & & $\mathbf{x}$ \\
\hline HC200 & GB093 & Área de colchón de pista & & & $\mathbf{x}$ & & \\
\hline HA780 & GB095 & Franja de pista & $\mathbf{x}$ & & & & \\
\hline HC210 & GB096 & Zona libre de obstáculos & & & $\mathbf{x}$ & & \\
\hline HD250 & GB161 & Área de deshielo de aronaves & & & & $\mathbf{x}$ & \\
\hline HE850 & GB162 & Punto de chequeo del servicio de radio navegación & & & & & $\mathbf{x}$ \\
\hline HE860 & GB163 & Comprobación de ubicación del altímetro & & & & & $\mathbf{x}$ \\
\hline HA790 & GB200 & Servicio de carreteras del aeródromo & $\mathbf{x}$ & & & & \\
\hline HC220 & GB220 & Obstáculo aeronáutico & & & $\mathbf{x}$ & & \\
\hline HA800 & GB225 & Zona libre de obstáculos OFZ & $\mathbf{x}$ & & & & \\
\hline HD260 & GB230 & Hangar de aviones & & & & $\mathbf{X}$ & \\
\hline HD270 & GB250 & Refugio de aviones & & & & $\mathbf{x}$ & \\
\hline HA810 & GB300 & Área de movimiento del aeródromo & $\mathbf{x}$ & & & & \\
\hline HC230 & GB480 & Sistema de indicadores de aproximación visual VASIS & & & $\mathbf{x}$ & & \\
\hline HC240 & GB485 & Sistema de luces de aproximación & & & $\mathbf{x}$ & & \\
\hline HD280 & GB810 & Servicio de tránsito aéreo & & & & $\mathbf{x}$ & \\
\hline HD290 & GB820 & Servicio de pasajeros del aeródromo & & & & $\mathbf{x}$ & \\
\hline HD300 & GB830 & Servicio en tierra para aeronaves & & & & $\mathbf{x}$ & \\
\hline
\end{tabular}

